\title{
Development and application of a new freckle criterion for technical remelting processes
}

\author{
B. Böttger ${ }^{1, a}$, G.J. Schmitz ${ }^{1}$, F.-J. Wahlers ${ }^{2}$, J. Klöwer ${ }^{2}$, J. Tewes ${ }^{2}$, and B. Gehrmann² \\ ${ }^{1}$ Access, Aachen, Germany \\ 2 Outokumpu VDM GmbH
}

\begin{abstract}
In technical solidification processes like Electro Slag (ESR) or Vacuum Arc Remelting (VAR), freckles present a serious type of defects which limit the maximum ingot size for many grades of steels and superalloys. Therefore, modelling of freckle formation is an important task for optimizing industrial remelting processes. Practically all present freckle models are based on a critical Rayleigh number. They are inspired by the "classical" assumption that freckles are caused by an inversion of the liquid density in the semisolid region. Plumes of the lighter segregated liquid evolve through perturbation of the metastable layering of the melt in the mushy zone, a mechanism which motivates its description via Rayleigh criteria. But these models are not suitable for materials like Alloy 718 which do not show a liquid density inversion, but nevertheless are prone to freckle formation in technical remelting processes. In this paper, a criterion is developed which - instead of using a Rayleigh number - is based on the evaluation of the non-isothermal component of an instantaneous down-hill flow of heavy segregated melt in a melt pool with axial symmetry. With knowledge of the exact pool geometry and the shape and properties of the mushy zone, the occurrence of freckles can be predicted. The model is applied to a technical ESR casting for which temperature fields and microstructural parameters have been obtained using CFD and 3D phase-field modelling, respectively. Furthermore, the implications are discussed which the new model offers for the understanding of freckles in technical remelting processes.
\end{abstract}

\section{Introduction}

Freckles have been the subject of intense research efforts and are in general considered to originate from convective instabilities during directional solidification. Much work has been devoted to clarify the mechanisms underlying such convective instabilities. Fundamental investigations on convective instabilities during directional solidification of binary alloys [1-5] are complemented by composition dependent models [6], numerical approaches [7], experiments with forced convection/high gravity [8-10] and by numerous observations and results for superalloys and steels in view of their technical importance [11-20].

For the prediction of freckle formation, many authors focus on the onset conditions for convective instabilities, e.g. $[21,22]$. Several of these approaches have also been extended for an inclined/tilted solidification front, e.g. [23, 24]. Here, with the assumption of different permeability values of the mushy zone parallel and perpendicular to the dendrite growth direction [25,26], the preferential formation of freckles at the mid-radius of ESR ingots has been explained [17,27]. An instructive recent overview about a variety of freckle models for superalloys is given in [17].

Almost all present model scenarios are based on the formulation of a Rayleigh criterion relating buoyant

\footnotetext{
${ }^{a}$ Corresponding author: b. boettger@access.rwth-aachen.de
}

driving forces for flow initialisation to stabilizing frictional forces. A mandatory pre-requisite for meaningfully applying such Rayleigh criteria thus is a density inversion being present in the liquid with a heavier - i.e. denser liquid located on top of a lighter - less dense - liquid. Liquids with a stable layering, meaning a dense, heavy liquid at the bottom, and a light liquid on the top, thus should not be prone to convection at all.

However, freckle type defects have also been observed for alloy 718 in industrial castings [12,20,27-29], although heavy elements like $\mathrm{Nb}$ lead to an increasing density of the segregated melt during solidification of this alloy which should result in the formation of a stable layering. Similar to buoyancy driven freckles, these defects appear as dark spots in the cross-section, preferentially at mid-radius of the ingot. The freckle regions reveal a composition as observed in the strongly enriched interdendritic regions [30-32] and frequently consist of a chain of small equiaxed grains [31,33].

In the scientific discussion, this contradiction has not been addressed for long time. The lack of density inversion for alloy 718 was either ignored [20,31] or rejected, pointing out that the high silicon contents of older grades of alloy 718 could have led to a decreasing density of the melt during solidification [12,27,30].

Eventually, based on the study of longitudinal sections through alloy 718 ingots, the real nature of this defect could be described as a type of centre segregates [27]

This is an Open Access article distributed under the terms of the Creative Commons Attribution License 4.0, which permits unrestricted use, distribution, and reproduction in any medium, provided the original work is properly cited. 


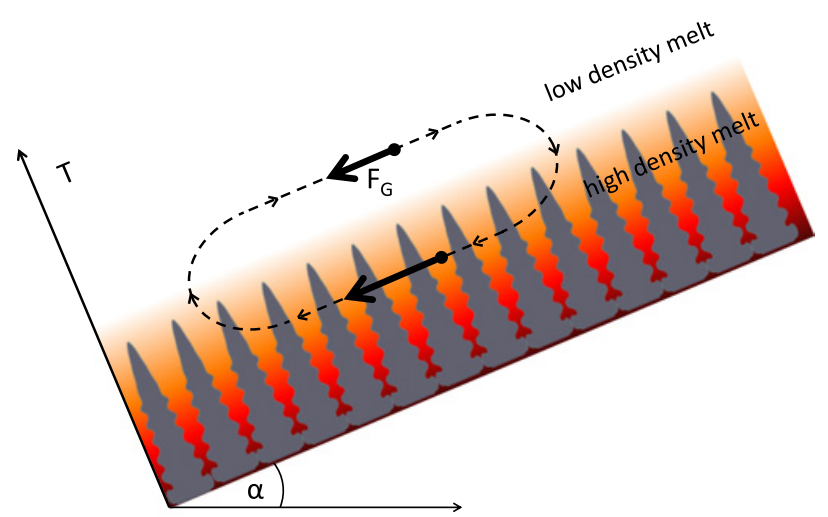

Figure 1. Schematic representation of a down-hill flow for a high density of the interdendritic liquid and a tilted solidification front (downflow freckles).

where heavy melt flows down along the pool surface. Due to the slight inclination of the channel with respect to the isotherm, the segregated melt gets in contact with already solidified material at higher temperature, and partial melting occurs.

Despite of these findings, Rayleigh number based criteria are still used to describe freckle initiation under those conditions [12,23,24,27,30,31], although the downhill flow of the heavy melt can be expected to be instantaneous, not requiring any initiation.

In the following, a new criterion for this type of channel segregates ("downflow freckles") is presented which recently has been discussed with respect to the general geometry of the melt pool [34]. It is also shown in this paper how the new approach can be applied to industrial remelting processes, and which type of supplementary simulation at the process and the microstructure scale is required in order to provide the fluid dynamical and geometrical data needed for modelling the relevant melt flow processes.

\section{Development of a new freckle criterion}

The important difference between downflow freckles and the classical case of a density inversion with upwards flowing plumes (buoyancy freckles) is the way how flow initiation occurs. For buoyancy freckles, at least if the solidification front is not tilted, there exists a priori no driving force for fluid flow (metastable situation). Considering a hypothetical cyclic flow path, the net force is 0 , and a perturbation of the liquid layers is necessary to initiate a cyclic flow (plume).

This is entirely different for materials like alloy 718 when the solidification front is tilted. As can be seen in Fig. 1, there is a driving force for a cyclic flow pattern which is associated with an instantaneous downhill flow of the heavier melt in the mushy zone and an uphill flow of the lighter liquid above the solidification front. It is obvious that no specific criterion is needed for the onset of such kind of flow.

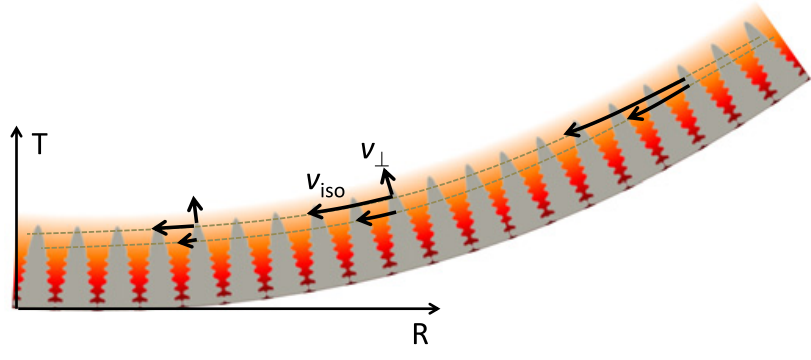

Figure 2. Origin of a flow component $v_{\perp}$ perpendicular to the isotherms in a curved melt pool.

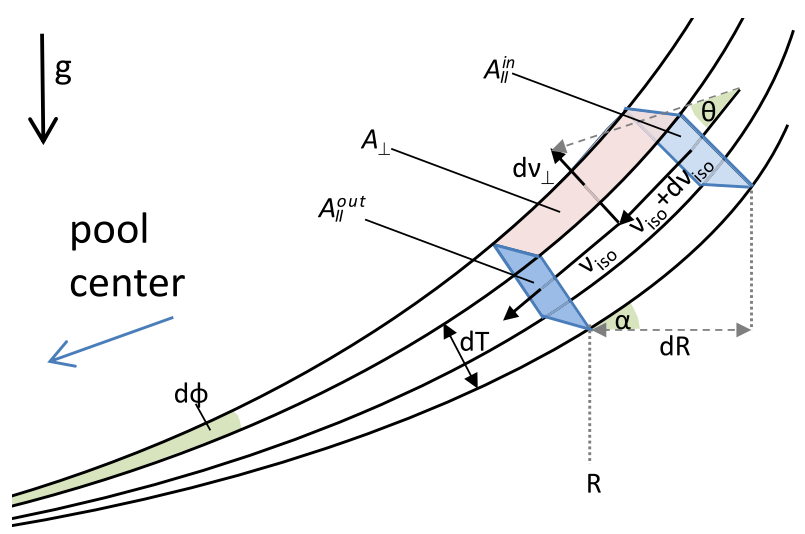

Figure 3. Flow balance in a differential volume element of the semisolid region.

Once the existence of an instantaneous downhill flow is accepted, it is straightforward to define a criterion for freckle formation: As long as the flow path essentially follows the isotherms, no melting will occur. But if for some reason - e.g. due to obstacles or due to a decreasing slope of the pool surface - the velocity of the downhill flow is reduced (Fig. 2), a flow component normal to the isotherms will arise. Melting of dendrite arms will occur if this perpendicular flow velocity exceeds the solidification velocity at this location (see below).

In the following it is assumed that, according to Darcy's law, the isothermal component of the downhill flow $v_{i s o}$ at temperature $T$ can be described as a function of the density difference $\varrho(T)-\varrho\left(T_{R e f}\right)$ with respect to the reference temperature $T_{R e f}$ (temperature of dendrite tips), the temperature dependent permeability $K(T)$, and the inclination angle $\alpha$ as follows:

$$
v_{\text {iso }}=K(T) \frac{\left[\varrho(T)-\varrho\left(T_{R e f}\right)\right] g}{\eta} \sin \alpha
$$

with $\eta$ being the dynamic viscosity of the melt, and $g$ the gravity constant.

If a melt pool with axial symmetry is assumed, the nonisothermal flux component $v_{\perp}$ can be derived considering a differential sector volume element with a thickness $d T$ resp. $d T / G$, where $G$ is the local temperature gradient (Fig. 3).

The differential flux change $d v_{\perp}$ can be obtained from the isothermal velocity change $d v_{i s o}$ and the cross sections $A_{/ /}^{\text {out }}$ and $A_{/ /}^{\text {in }}$ for the isothermal flow and $A_{\perp}$ 
for the non-isothermal flow component from continuity considerations

$$
\begin{aligned}
\mathrm{A}_{\perp} \mathrm{dv}_{\perp} & =\mathrm{A}_{\|}^{\text {out }}\left(\mathrm{v}_{\text {iso }}+\mathrm{dv}_{\text {iso }}\right)+\mathrm{A}_{\|}^{\text {in }} \mathrm{v}_{\text {iso }} \\
\text { with } \quad \mathrm{A}_{\|}^{\text {out }} & =\frac{\mathrm{d} \varphi(\mathrm{R}+\mathrm{dR})}{2 \pi} \frac{\mathrm{dT}}{\mathrm{G}+\mathrm{dG}}, \\
\mathrm{A}_{\|}^{\text {in }} & =\frac{\mathrm{d} \varphi \mathrm{R}}{2 \pi} \frac{\mathrm{dT}}{\mathrm{G}} \\
\mathrm{A}_{\perp} & =\frac{\mathrm{d} \varphi \mathrm{R}}{2 \pi} \frac{\mathrm{dR}}{\cos \alpha}
\end{aligned}
$$

the differential non-isothermal flux perpendicular to the temperature gradient can be calculated. $d \varphi$ represents the angular section of the rotationally symmetric pool profile and is of no further importance and eliminates in the further procedures.

Assuming $d G \ll G$ and $d v_{i s o} d R \ll v_{i s o} R+v_{i s o} d R+$ $R d v_{i s o}$, and integrating $d v_{\perp}$ between the solidus temperature $T_{S}$ and a given position in the mushy zone at temperature $T^{*}$ provides the value of the normal flow component at this position [34]:

$$
\begin{aligned}
& v_{\perp}\left(T^{*}, R\right)=\frac{1}{f_{L}\left(T^{*}\right)} \int_{T_{S}}^{T^{*}} f_{L}(T) d v_{\perp}= \\
& \frac{1}{f_{L}\left(T^{*}\right)} \int_{T_{S}}^{T_{S}^{*}} \frac{f_{L}}{G}\left[-\frac{v_{i s o} d G}{G d R}+\frac{v_{i s o}}{R}+\frac{d v_{i s o}}{d R}\right] \cos \alpha d T .
\end{aligned}
$$

This formulation for $v_{\perp}$ comprises three contributions, Eqs. ((4)-(6)), which can be attributed to the effects of a variation of $G, R$ and $v_{\text {iso }}$ along the flow path:

$$
\begin{array}{r}
\mathrm{v}_{\perp, \mathrm{I}}\left(\mathrm{T}^{*}, \mathrm{R}\right)=\frac{1}{\mathrm{f}_{\mathrm{L}}\left(\mathrm{T}^{*}\right)} \int_{\mathrm{T}_{\mathrm{S}}}^{\mathrm{T}^{*}} \frac{\mathrm{f}_{\mathrm{L}}}{\mathrm{G}} \frac{\mathrm{dv_{ \text {iso } }}}{\mathrm{dR}} \cos \alpha \mathrm{dT} \\
\mathrm{v}_{\perp, \mathrm{II}}\left(\mathrm{T}^{*}, \mathrm{R}\right)=\frac{1}{\mathrm{f}_{\mathrm{L}}\left(\mathrm{T}^{*}\right)} \int_{\mathrm{T}_{\mathrm{S}}}^{\mathrm{T}^{*}} \frac{\mathrm{f}_{\mathrm{L}}}{\mathrm{G}} \frac{\mathrm{v}_{\text {iso }}}{\mathrm{R}} \cos \alpha \mathrm{dT} \\
\mathrm{v}_{\perp, \mathrm{III}}\left(\mathrm{T}^{*}, \mathrm{R}\right)=\frac{1}{\mathrm{f}_{\mathrm{L}}\left(\mathrm{T}^{*}\right)} \int_{\mathrm{T}_{\mathrm{S}}}^{\mathrm{T}^{*}}-\frac{\mathrm{f}_{\mathrm{L}}}{\mathrm{G}} \frac{\mathrm{v}_{\text {iso }} \mathrm{dG}}{\mathrm{GdR}} \cos \alpha \mathrm{dT} .
\end{array}
$$

Once the value of $v_{\perp}\left(T^{*}, R\right)$ is known all over the melt pool, a simple criterion for freckle formation can be derived according to Flemings [35]: If at any location through the mushy zone the local value of $v_{\perp}$ exceeds the local solidification velocity $T / G_{\perp}$, freckle formation must be expected, as the upward flow of segregated melt in this case starts to dissolve dendrite arms or has a negative impact on the growth of the dendrite tips. As a consequence, the permeability of the mushy zone increases, and the local flow pattern thus is further fostered.

The following sharp criterion for downflow freckle formation can thus be formulated:

$$
C_{\mathrm{F}}(\mathrm{R})=\max \left(v_{\perp}\left(T^{*}, R\right) \frac{G_{\perp}\left(T^{*}, R\right)}{T^{\prime \prime}}\right)>1 .
$$

The maximum ( $\max$ ) here corresponds to the temperature $T^{*}$ in the mushy zone, where the maximum value of the argument is reached.

However, there is a further restriction for the maximum values of $v_{\perp}$ : The effective flow direction, corresponding

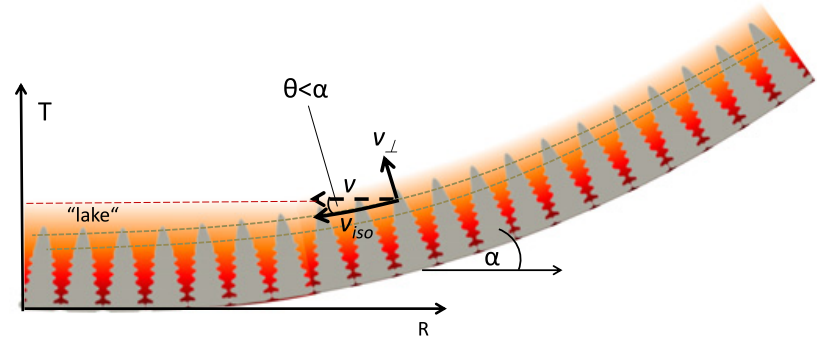

Figure 4. Condition for "lake" formation.

to a vector addition of the isothermal flow component $v_{i s o}$ and the non-isothermal flow component $v_{\perp}$, can never be directed upwards (i.e. $\vartheta<\alpha$, see Fig. 4), because there is no driving force for the heavy melt to move against the gravity vector. This restriction is most important close to the centre of the melt pool, where, due to the axial symmetry, the heavy melt is converging. Without this additional restriction, unphysical high values of $v_{\perp}$ would be predicted there. Instead, a lake of heavy melt is forming, and the flow direction is forced to be horizontal (Fig. 4). As a consequence, the condition

$$
v_{\perp}\left(T^{*}, R\right)<v_{\text {iso }}\left(T^{*}\right) \tan \alpha\left(T^{*}\right)
$$

has to be applied before calculating $C_{\mathrm{F}}(R)$ according to Eq. (7).

\section{Application to industrial ESR casting}

The new freckle criterion for downflow freckles derived in Section 2 is a priori based on theoretical arguments. In contrary to classical Rayleigh number based criteria being typically calculated as function of the local tilt angle $\alpha$ and the local thermal gradient $G$, the new criterion depends on the local changes (derivatives) of $\alpha$ (Eq. (4)) and $G$ (Eq. (6)). As a consequence, freckle prediction requires much more detailed knowledge about the actual shape of the pool profile and the individual isotherms, which is difficult to obtain experimentally.

Thus, numerical simulation methods are required. For application of the new freckle approach to an ESR casting of alloy 718 with a block diameter of $0.5 \mathrm{~m}$, a computational fluid dynamics (CFD) model has been developed. The two commercial codes ANSYS FLUENT and ANSYS EMAG were coupled to solve Reynolds-averaged Navier-Stokes equations, heat transfer and Maxwell's equations [36]. Exemplarily, the stationary temperature distribution obtained for a smaller test casting is shown in Fig. 5.

In order to obtain smooth isotherms, also for the first and second derivative, $10^{\text {th }}$ order polynomials were calculated based on the numerical results and used as input for the freckle model, as shown in Fig. 6. Note that much smaller temperature steps $(0.1 \mathrm{~K})$ between the isotherms have been used for reasons of numerical accuracy.

Apart from the geometry of the isotherms, evaluation of the freckle criterion requires knowledge of the permeability of the semisolid region $K$, the liquid density $\varrho$, (Eq. (1)) as well as of the fraction of liquid (Eq. (3)), 


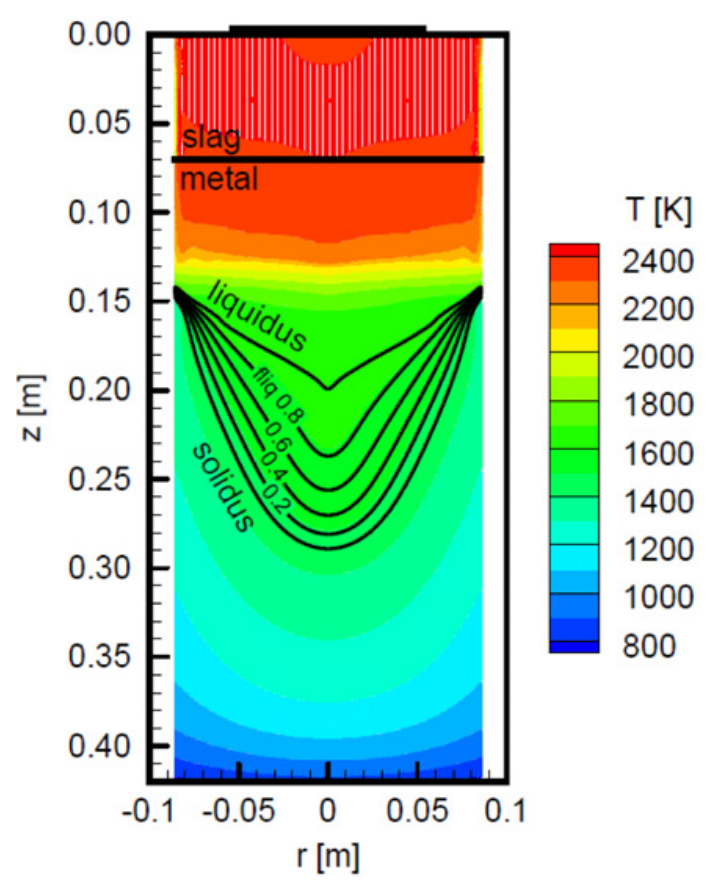

Figure 5. Stationary temperature distribution in an ESR casting with a diameter of $0.172 \mathrm{~m}$ (with kind permission of [36]).

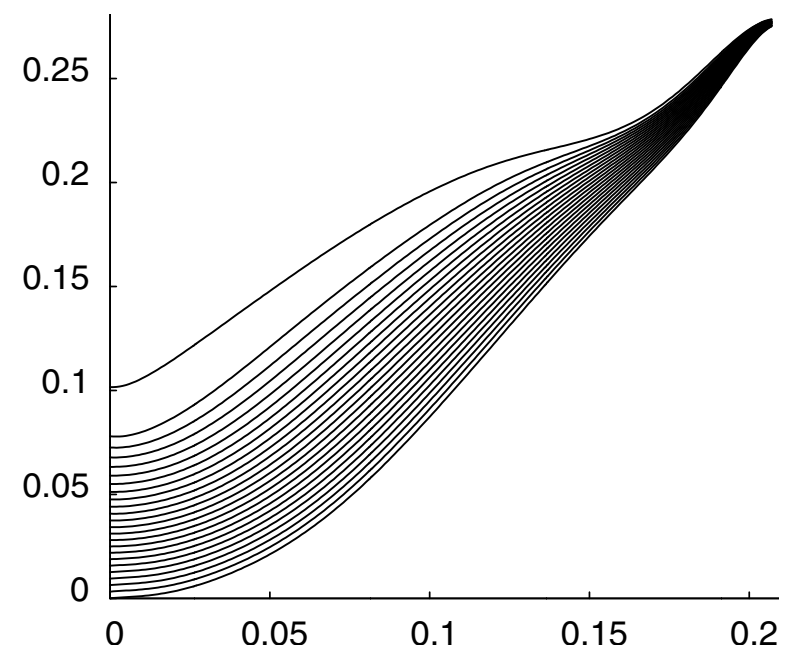

Figure 6. Shape of isotherms for the ESR casting, at $T_{L i q}$ and below $(\Delta T=2 \mathrm{~K}$, dimensions in $\mathrm{m})$.

all three as function of temperature. These functions have been obtained based on 3D phase-field modelling using the commercial software MICRESS ${ }^{\circledR}$ [37]. The multicomponent phase-field simulation was online-coupled to thermodynamic and diffusion databases [38] and took intermetallic phases into account like $\mathrm{Ti}(\mathrm{C}, \mathrm{N})$ which forms at higher temperature in front of the dendrites (Fig. 7). Especially the knowledge of the exact dendrite geometry and the respective $f_{L}-T$ relation is crucial because they strongly differ from what can be obtained from simple solidification models, especially in the region of the dendrite tip.

The fraction solid as obtained by averaging the results of many time steps of the phase-field simulation with subsequent smoothing is shown in Fig. 8. In a first step,
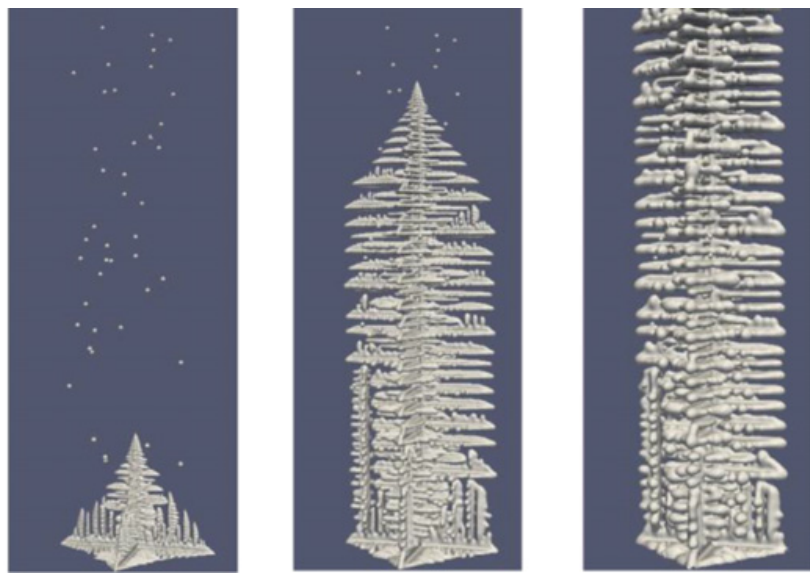

Figure 7. 3D microstructure simulation of 3D dendritic growth of alloy 718 using the phase-field code MICRESS ${ }^{\circledR}[37,41]$.

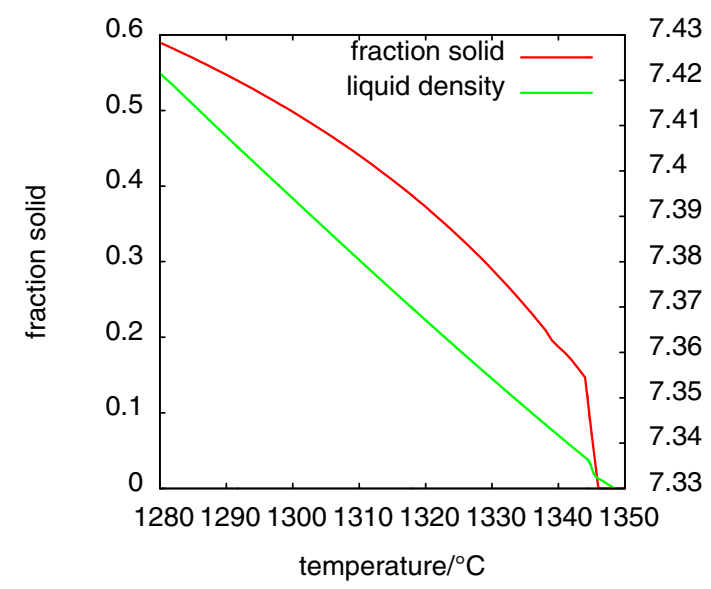

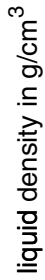

Figure 8. Fraction solid and liquid density as functions of temperature, as obtained by MICRESS ${ }^{\circledR}$ simulation.

the dynamic permeability in isothermal direction was calculated using the Blake-Kozeny-relation as a function of the fraction liquid [43]:

$$
\mathrm{K}=0.0649+0.0543\left[\frac{f_{l}}{1-f_{l}}\right]^{0.25} \lambda^{2}
$$

In future, the permeability will be directly calculated based on the 3D microstructure by use of CFD.

The average melt density was calculated from the liquid compositions obtained from the MICRESS ${ }^{\mathbb{R}}$ simulations using the molar volume averaging approach used by Sung et al. [39]. The resulting density curve is also plotted in Fig. 8. The dynamic viscosity $\eta$ was taken from literature [40].

After having collected all the required parameters, the freckle criterion according to Eq. (7) has been calculated using a Python script. The results can be seen in Fig. 9 as a function of the block radius. Two maxima are found, one pronounced around $\mathrm{r}=0.04 \mathrm{~m}$, and a smaller one at $\mathrm{r}=0.17 \mathrm{~m}$. Careful analysis under consideration of the individual contributions (Eqs. (4)-(6)) show that besides the steepness especially the curvature of the isotherms play 


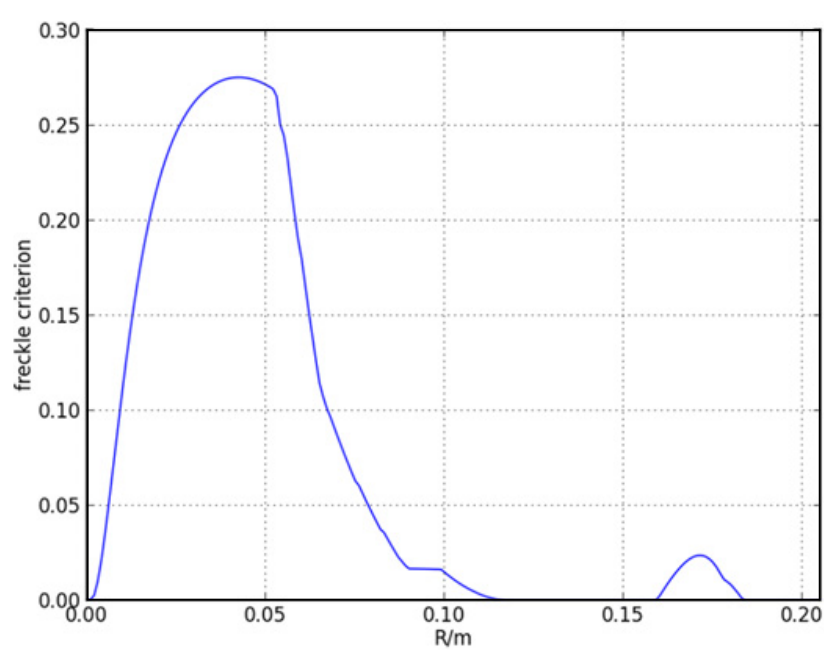

Figure 9. Predicted freckle criterion $\mathrm{C}_{\mathrm{F}}$ (Eq. (7)) as function of the block radius.

an important role for the exact position of the maxima of $\mathrm{C}_{\mathrm{F}}$, as has been shown in a parameter study [34].

In accordance with the sharp condition $\mathrm{C}_{\mathrm{F}}>1$ formulated in Eq. (7), the results in Fig. 9 indicate that no freckles are expected, which coincides with the fact that also no freckles have been found yet for the given process conditions. But the results also imply that the highest probability for freckles is located close to the centre.

\section{Discussion}

The application of the newly developed freckle criterion to a technical ESR casting has shown that it can be very helpful for understanding freckle formation in complex ESR or VAR remelting processes, especially for alloys without liquid density inversion. The criterion is sharply defined $\left(C_{\mathrm{F}}>1\right)$, thus not requiring any calibration. Rayleigh number based criteria, in contrast, require an experimental calibration of the critical Rayleigh number which may even be not be identical for different types of alloys [42]. Furthermore, they seem to be less powerful in case of strongly curved pool profiles like in ESR or VAR [27].

An analysis at which temperature the highest contributions to $C_{\mathrm{F}}$ are observed (Eq. (7)) leads to the conclusion that downflow freckles form at high liquid fractions $(\sim 85-90 \%)$, i.e. in the upper part of the mushy zone close to the dendrite tips. The main reason is the strong decrease of the permeability in the mushy zone for a liquid flowing perpendicular to the dendrite growth direction (i.e. parallel to the isotherms) according to the Blake-Kozeny relation (Eq. (8)). This seems to be in conflict with measurements of the freckle composition in alloy 718 implying that freckles are fed by a melt originating from a region in the mushy zone with a fraction liquid of about 0.4-0.6 [30,43]. However, it can be assumed that, even if freckles are initiated at considerably higher liquid fractions, they will erode the dendritic network, locally increase permeability, and thus penetrate downward deeper into the mushy zone. Also for classical buoyancy freckles an initiation at high $f_{L}$ values has been shown by means of numerical simulations [42].

Furthermore, the question arises whether the new understanding of downflow freckle formation presented in this paper might have as well implications for classical buoyancy freckles in ESR or VAR ingots. Even in case of an existing liquid density inversion, the argument of an instantaneous onset of fluid flow should still be valid. The resulting isothermal flow pattern, which in this case would be in uphill direction, could also lead to freckle formation or at least help to induce convective instabilities. Such an effect has been demonstrated by numerical simulation for a tilted casting and was interpreted in terms of a reduction of the critical Rayleigh number due to inclination [42]. The model equations which have been derived in this paper for downflow freckles could at least indicate their preferential locations.

A further consequence of the model is that, without any inclination, freckle formation should be impossible at any local Rayleigh number, as long as the liquid density is increasing with decreasing temperature in a "stable layering". This is evident as all three contributions to a possible non-isothermal flow $v_{\perp}$ (Eqs. (4)-(6)) vanish if $v_{i s o}$ is zero. But to reduce the freckle risk, the pool shape should not only be as flat as possible: In contrast to the classical criteria, the change of inclination, i.e. the curvature of the pool profile, or more exactly $d \cos (\alpha) / d R$, is essential and should be reduced in order to minimize term I of $v_{\perp}$ (Eq. (4)). At the same time, according to Eq. (6), an increasing gradient along the pool radius can help to avoid freckles, if this increase is located appropriately.

As a possible extension of this model, macroscopic flow pattern due to thermal convection [44] or induction could be included by adding the local velocity values to $v_{i s o}$. The result would be a locally increased or decreased risk for freckle formation, depending on the macroscopic flow direction. Further possible extensions relate to the consideration of contributions to $v_{\perp}$ originating from flow evoked by density discontinuities between solid and liquid.

\section{Conclusions and outlook}

An entirely new approach to freckle formation during ESR or VAR of alloys without liquid density inversion like alloy 718 has been developed. Unlike in most classical freckle criteria, no Rayleigh number is used. Instead, the present approach is based on the evaluation of the non-isothermal component of an instantaneous flow of heavy melt down towards the centre of the melt pool. This new approach allows for the definition of a sharp criterion for freckle formation which is based on the pool shape (geometry of the isotherms), the density gradient in the melt, the permeability of the mushy zone and the solidification rate.

Furthermore, application to a real industrial remelting process has been exemplarily demonstrated. In order to provide the needed input data for the model, 
CFD modelling on the process scale as well as 3D microstructure modelling on the dendrite scale are indispensable.

For further validation, the presented methodology will be applied to industrial ESR or VAR castings where the formation of freckles is observed and their preferential locations in the block, and the corresponding process conditions could be successfully determined.

\section{References}

[1] A.C. Fowler, IAM Journal of Appl. Mathematics 35(2) 159 (1985)

[2] G. Amberg, G.M. Homsy, Journal of Fluid Mechanics 25279 (1993)

[3] S.V. Garimella, J.P. McNulty, L.Z. Schlitz, Metall. Mater. Trans. A 26(4) 971 (1995)

[4] S.D. Felicelli, J.C. Heinrich, D.R. Poirier, J. Cryst. Growth 191(4) 879 (1998)

[5] C. Frueh, D.R. Poirier, S.D. Felicelli, Mat. Sci. and Eng. A 328(2) 245 (2002)

[6] J. Rösler et al., Advanced Engineering Materials 5(7) 469 (2003)

[7] S.D. Felicelli, D.R. Poirier, J.C. Heinrich, Metall. Mater. Trans. B 29(4) 847 (1998)

[8] D.N. Riahi, J. Cryst. Growth 226(3) 393 (2001)

[9] D.G. Neislon, F.P. Incropera, Int. J. of Heat and Mass Trans. 36(2) 489 (1993)

[10] G. Quillet et al. Modeling of Casting, Welding and Advanced Solidification Processes X 253 (2003)

[11] L. Wang, B.Y. Zhong, J.X. Dong, M.C Zhang, Rare Metal Materials and Engineering 36(12) 2104 (2007)

[12] P. Auburtin, T. Wang, S.L. Cockcroft, A. Mitchell, Metall. Mater. Trans. B 31(4) 801 (2000)

[13] J.P. Gu, C. Beckermann, A.F. Giamei, Metall. Mater. Trans. A 28(7) 1533 (1997)

[14] S.T. Wlodeck, R.D. Field, Superalloys 718, 625, 706 and various derivatives 167-176 (1994)

[15] M.C. Schneider et al. Metall. Mater. Trans. A 28(7) 1517 (1997)

[16] H.F. Cao, H.F. Shen, B.C. Liu, Rare Metal Materials and Engineering 35(12) 1849 (2006)

[17] J. Valdes, P. King, X.B. Liu, Metall. Mater. Trans. A 41(8) 2408 (2010)

[18] S. Tin, T.M. Pollock, Journal of Materials Science 39(24) 7199 (2004)

[19] Z.D. Long, W.H. Yang, K.M. Chang, Superalloys $718,625,706$ and various derivatives 745 (2001)
[20] K. Morita, T. Suzuki., T. Taketsuru, D.G. Evans, W. Yang, Superalloys 718, 625, 706 and various derivatives 149 (2001)

[21] J.C. Ramirez, C. Beckermann, Metall. Mater. Trans. A 34(7) 1525 (2003)

[22] W.H. Yang et al., Metall. Mater. Trans. A 322397 (2001)

[23] W.H. Yang et al., JOM 56(8) 56 (2004)

[24] C.F. Baker, D.N. Riahi, Centrifugal Materials Processing 163 (1997)

[25] R.G. Erdmann, D.R. Porier, A.G. Hendrick, Materials Science Forum 649399 (2010)

[26] D.R. Poirier, Met Trans B 18(1) 245 (1987)

[27] P. Auburtin, S.L. Cockcroft, A. Mitchell, A.J. Schmalz, Superalloys 718, 625, 706 and Various Derivatives, TMS 47 (1997)

[28] J.A. Van den Avyle, J.A. Brooks, A.C. Powell, JOM 50(3) 22 (1998)

[29] R. Kennedy et al., Advanced Technologies for Superalloy affordability TMS 159 (2000)

[30] P. Auburtin, S.L. Cockcroft, A. Mitchell, Superalloys 1996, 443-450 (1996)

[31] W.H. Yang et al., Superalloys 718, 625, 706 and various derivatives 113 (2001)

[32] K.O. Yu et al., Journal of Metals 146 (1986)

[33] A.F. Giamei, B.H. Kear, Metallurgical Transactions 1(7) 2185 (1970)

[34] B. Böttger, G. J. Schmitz, F-J. Wahlers, J. Klöwer, J. Tewes, B. Gehrmann, High Temperatures-High Pressures 42(2), 115 (2013)

[35] M.C. Flemings, G.E. Nereo, Trans TMS-AIME 239 1449 (1967)

[36] N. Giesselmann, A. Rückert, H. Pfeifer, J. Tewes, J. Klöwer, 1st International Conference on Ingot Casting, Rolling and Forging (ICRF), 3-7 June 2012 Aachen

[37] http://www.micress.de

[38] Themo-Calc Software, http://www.thermocalc.se

[39] P.K. Sung, D.R. Poirier, E. McBridge, Mater. Sci. Eng. A 231, 189 (1997)

[40] J. Guo, M.T. Samonds, J. Phase Equilib. Diffus. 28, 58 (2006)

[41] B. Böttger 2013, unpublished

[42] C. Beckermann, J.P. Gu J, W.J. Boettinger, Metall. Mater. Trans. A, 312545 (2000)

[43] J. Madison et al., Metall. Mater. Trans. A, 43369 (2012)

[44] V. Sahai, R.A. Overfelt, P. Banerjee, Superalloys 718, 625, 706 and various derivatives 107 (1996) 